\title{
Uso de óleo na dieta de eqüinos submetidos ao exercício
}

\author{
Fernando Mattos ${ }^{1}$, Kleber Villela Araújo ${ }^{2}$, Gilberto Gonçalves Leite ${ }^{3}$, Herbert de Moura Goulart ${ }^{4}$ \\ 1 União Educacional do Planalto Central - UNIPLAC - Brasília - DF. \\ 2 Ministério da Agricultura, Pecuária e Abastecimento - Brasília - DF. \\ ${ }^{3}$ Embrapa Cerrados - CPAC - Brasilia - DF. \\ ${ }^{4}$ RBMont - Brasília - DF.
}

RESUMO - Objetivou-se com esta pesquisa avaliar, por meio de parâmetros hematofisiológicos, o desempenho atlético de cavalos submetidos a exercícios de média intensidade alimentados com dietas suplementadas com dois níveis de óleo vegetal (250 e $500 \mathrm{~g}$ /dia). Foram utilizados 18 eqüinos machos, castrados, sem raça definida, com faixa etária entre 4 e 8 anos de idade e peso corporal de $456 \pm 2,90 \mathrm{~kg}$. Os animais foram distribuídos em um delineamento inteiramente aleatório, em um esquema de parcela subdividida no tempo, em que os tempos de coleta constituíram a subparcela e os níveis de óleo de soja (0, 250 e $500 \mathrm{~g} / \mathrm{cavalo/dia),}$ a parcela. Os cavalos receberam, durante 30 dias, 8,40; 7,95 e 7,30 kg/dia das rações contendo 0,250 e $500 \mathrm{~g}$ de óleo, respectivamente, divididas em três refeições diárias. Ao final dos 30 dias, foi realizado o exercício-teste com todos os cavalos, durante 2 horas, ao trote alongado. Foram avaliados, no início e final do exercício, os teores de glicose e lactato sangüíneos, o hematócrito e a temperatura corporal. As freqüências cardíaca e respiratória foram tomadas no início, 1 e 2 horas de exercício e 15 minutos após o exercício. Verificou-se efeito da interação tempos de coleta $\times$ níveis de óleo na dieta sobre os teores de glicose e lactato e valores de hematócrito, temperatura corporal, freqüência cardíaca e respiratória. A adição de $250 \mathrm{e} 500 \mathrm{~g}$ de óleo na dieta de eqüinos submetidos ao exercício de média intensidade proporcionou melhora no desempenho hematofisiológico e, portanto, pode resultar em melhor desempenho atlético dos cavalos. Os cavalos que consumiram óleo na proporção de $500 \mathrm{~g} /$ dia apresentaram melhor recuperação pós-prova, confirmada pela freqüência cardíaca e pelo hematócrito.

Palavras-chave: freqüência cardíaca, glicose, hematócrito, lactato

\section{Use of oil in diet of equine under exercise}

\begin{abstract}
The objective of this research was to estimate, by haematophysiological parameters, the physical performance of horses under exercise of mid intensity and fed diets with two levels of vegetal oil (250 and $500 \mathrm{~g} / \mathrm{day})$. Eighteen castrated thoroughbred horses aging from 4 and 8 years old and averaging body weight of $456 \pm 2.90 \mathrm{~kg}$ were alloted to a split plot approach with repeated measures over time in a completely randomized design, with the collection times in the sub-plots and the soybean oil levels $(0,250$ and $500 \mathrm{~g}$ per horse per day) in the plots. During 30 days, the horses were fed 8.40, 7.95, and $7.30 \mathrm{~kg} /$ day of diets with 0,250 and $500 \mathrm{~g}$ of oil, respectively, divided in three daily feeds. At the end of 30 days, the exercise test was performed with all horses, during 2 hours, at large trot. At the beginning and at the end of the exercise, the levels of glucose, lactate, haematocrit, body temperature were estimated. The heart and respiratory rates were taken at the beginning, 1 and 2 hours of exercise and 15 minutes after the end of the exercise. Interaction of collection times $\times$ dietary oil levels on the levels of glucose, lactate, and haematocrit, body temperature, and heart and respiratory frequencies was observed. The supplementation of 250 and $500 \mathrm{~g}$ of oil in the diet of equines under exercise of mid intensity increased haematophysiological performance, that can improve horse athletic performance. The horses daily fed $500 \mathrm{~g}$ of oil showed better recovery post-trial, and that is verified by the values of heart rate and of haematocrit.
\end{abstract}

Key Words: glucose, head rate, haematocrit, lactate

\section{Introdução}

Para obtenção de máximo desempenho produtivo dos eqüinos no esporte ou em trabalhos, são necessários conhecimentos que contribuam para retardar o início da fadiga muscular. As causas exatas da fadiga muscular não estão bem definidas, entretanto, acredita-se que fatores como baixo nível de glicose sangüínea, esgotamento do glicogênio muscular, aumento de lactato, acúmulo de $\mathrm{NH}_{3}$ na célula, perda de adenosina trifosfato (ATP) muscular e eletrólitos conduzem à fadiga (Hiney \& Potter, 1996).

A introdução de gordura na dieta de animais atletas ocorreu em 1973, com o objetivo de prevenir a rabdomiólise em cães de corrida (Kronfeld et al., 1998). A partir desta data, 
estudos com cavalos também foram desenvolvidos com o intuito de prevenir essa patologia. Posteriormente, a inclusão de gordura na dieta de eqüinos atletas começou a ser estudada com o objetivo de reduzir a fadiga muscular. O possível retardo da fadiga muscular, obtido com a adição de gordura na dieta de cavalos atletas, pode significar a manutenção da velocidade de exercício por períodos mais longos ou o aumento da velocidade do exercício (Meyer et al., 1989).

Segundo Lawrence et al. (1995), a gordura pode ser adicionada em dietas para eqüinos para aumentar sua densidade energética. Além desse fato, Frape (1994) comenta que dietas contendo gordura reduzem os problemas de cólica e laminite nos eqüinos e promovem o metabolismo de lipídeos no fígado e no músculo. Além disso, não sofrem fermentação microbiana e favorecem a menor produção de $\mathrm{CO}_{2}$ por mol de ATP gerado. A inclusão de gordura na dieta pode favorecer o desempenho de cavalos exercitados em regiões de clima quente, por diminuir o incremento calórico, visto que aproximadamente $3 \%$ a mais de calor é produzido durante a formação de ATP via oxidação da glicose, quando comparado à oxidação dos ácidos graxos (Konh et al., 1996).

Segundo Cunha (1991), o treinamento tem demonstrado aumentar a eficiência na utilização de gordura como fonte de energia. Scott et al. (1992) demonstraram que a adição de $10 \%$ de gordura animal a uma dieta concentrada por três semanas resultou em aumento e uso das reservas de glicogênio muscular em animais puro-sangue Ingleses submetidos a exercício intenso com condições corporais média e média alta. De acordo com pesquisas, cavalos treinados e adaptados a dietas suplementadas (de 21 dias a 8 meses) com gordura aumentam o metabolismo lipídico, ou seja, apresentam maior capacidade de oxidar ácidos graxos para utilizá-los como fonte de energia, poupando as reservas de glicogênio (Jones et al., 1992; Meyer et al., 1989; Oldham et al., 1990; Scott et al., 1992; Harkins et al., 1992). Ao mesmo tempo, tem-se verificado aumento na concentração de lactato após o exercício em cavalos alimentados com dietas contendo gordura (Ferrante et al., 1994; Taylor et al., 1995; Oldham et al., 1990). Segundo Taylor et al. (1995), o aumento na oxidação de ácidos graxos eleva a produção de acetil-CoA, inibindo a enzima piruvato desidrogenase e, com isso, aumenta a concentração de piruvato. Este piruvato "sobressalente" é convertido em lactato em condições de deficiência de oxigênio.

A literatura tem apresentado alguns resultados divergentes quanto ao uso de gordura na ração de cavalos atletas. Acredita-se que essas divergências são decorrentes das diferenças na quantidade e no tipo de gordura adicionada, da composição da dieta basal, do tempo de suplementação, da condição corporal do cavalo, do regime de treinamento e da intensidade do exercício-teste (Hiney \& Potter, 1996).

Ainda não está definido quanto tempo antes da competição deve ser iniciada a alimentação com uma dieta contendo gordura, nem por quanto tempo essa suplementação deve ser mantida. É recomendado que a gordura seja consumida pelo menos 6 a 11 semanas antes de qualquer evento para que haja adaptação enzimática e metabólica (Frape, 1994). Em alguns experimentos nos quais foi avaliada a resposta metabólica de cavalos em exercício sob suplementação com gordura na dieta, os autores adotaram o período de 21 dias (Meyer et al., 1989; Oldham et al., 1990; Scott et al., 1992).

A gordura adicionada em níveis acima de $20 \%$ nas dietas não tem apresentado efeito negativo sobre o consumo ou a digestibilidade de matéria seca e energia em cavalos (Hiney \& Potter, 1996). Ainda não se tem definida a quantidade exata de gordura a ser suplementada, mas na literatura são relatados níveis de inclusão de 10 a $12 \%$ do total da dieta, 10 a $12 \%$ do concentrado e 12 a $20 \%$ da energia digestível da dieta (Hiney \& Potter, 1996). Konh et al. (1996) recomendam 250 a $500 \mathrm{~g}$ de óleo vegetal/dia/cavalo, entretanto, Hintz (1997), em revisão, afirma que um cavalo deve consumir diariamente, no mínimo, $500 \mathrm{~g}$ de gordura para apresentar os efeitos metabólicos desejados, observando-se que esta quantidade deve ser aumentada gradualmente até $1.000 \mathrm{~g}$ para máximo efeito.

Objetivou-se com esta pesquisa avaliar, por meio de parâmetros hematofisiológicos, o desempenho atlético de cavalos alimentados com dietas contendo dois níveis de óleo de soja ( 250 e $500 \mathrm{~g}$ por dia) submetidos a exercícios de média intensidade.

\section{Material e Métodos}

O experimento foi realizado durante os meses de fevereiro e março de 2003 no 3o Esquadrão de Polícia Montada de Brasília, no Distrito Federal. Foram utilizados 18 cavalos machos, castrados, sem raça definida, com 4 a 8 anos de idade e peso corporal de $456,4 \pm 2,90 \mathrm{~kg}$. Os animais foram submetidos a exames físicos e bucais, sendo desverminados com solução de ivermectina a $1 \%$ e alojados em baias individuais contendo água e sal mineral à vontade para o controle diário da ingestão das dietas experimentais.

Os tratamentos consistiram da adição de três níveis de óleo de soja $(0,250$ e $500 \mathrm{~g} /$ animal/dia) na dieta, combinados com diferentes tempos de coleta de sangue e mensuração 
de parâmetros fisiológicos. Os parâmetros glicose e lactato, hematócrito e temperatura retal foram avaliados em dois tempos (antes e após o exercício-teste), enquanto as freqüências cardíaca e respiratória foram tomadas em quatro tempos (antes do início, após 1 e 2 horas de exercício e 15 minutos após o exercício). Os animais foram distribuídos em um delineamento inteiramente aleatório, com seis repetições, em um esquema de parcelas subdivididas, em que os tempos de coleta e/ou as mensurações dos parâmetros sangüíneos e fisiológicos constituíram a subparcela e os níveis de óleo de soja, a parcela.

As dietas foram formuladas utilizando-se concentrado comercial, farelo e óleo de soja e feno de coastcross (Tabela 1), visando atender $100 \%$ das exigências nutricionais de um eqüino adulto com peso médio de $450 \mathrm{~kg}$ submetido a trabalho ou exercício de média intensidade, segundo o NRC (1989). Os componentes da dieta eram pesados e imediatamente adicionados e misturados no cocho dos animais. Os cavalos passaram por um período de adaptação à dieta (uma semana), no qual os níveis de óleo foram aumentados lentamente ( $15 \%$ diariamente) até os valores estipulados. Posteriormente, foram alimentados três vezes por dia $(6,12 \mathrm{e} 17 \mathrm{~h})$, durante 30 dias, com as dietas experimentais, nas quantidades de 8,40; 7,95 e 7,30 kg das dietas contendo 0,250 e $500 \mathrm{~g}$ de óleo, respectivamente (Tabela 1). Estas quantidades foram calculadas com base nas análises químicas dos componentes da dieta, de forma a equiparar entre os tratamentos o consumo de proteína bruta (PB), energia digestível (ED) e a relação concentrado:volumoso (Tabela 1).

O concentrado comercial peletizado foi composto de milho moído, glúten de milho, farelo de arroz, farelo de soja, farelo de trigo, melaço em pó, feno de alfafa, calcário, fosfato bicálcico, sal comum e suplemento mineral e vitamínico (Tabela 2).

Durante os 30 dias experimentais, os cavalos foram condicionados fisicamente segundo a rotina normal da Polícia Montada de Brasília, que constou de 6 horas diárias de patrulha por cinco dias semanais, alternando os andamentos em trote, passo e momentos de parada. Ao final dos 30 dias, foi executado o exercício-teste com todos os cavalos ao mesmo tempo, em um terreno plano, durante 2 horas (12 às 14h), mantendo os animais sempre no trote alongado e na mesma seqüência. Neste dia, os animais não receberam a alimentação de $12 \mathrm{~h}$ para que não ocorressem possíveis influências nos parâmetros avaliados (Pagan, 1999).

Antes do início e após o exercício-teste, procedeu-se à coleta de amostra de sangue na veia jugular esquerda no seu terço médio, utilizando-se tubo a vácuo, modelo
Tabela 1 - Consumo diário dos componentes e dos nutrientes das dietas experimentais, com base na matéria natural

Table 1 - Daily feed intake of the components and nutrients of the experimental diets, as-fed basis

\begin{tabular}{|c|c|c|c|}
\hline \multirow[t]{2}{*}{$\begin{array}{l}\text { Component } \\
\text { Component }\end{array}$} & \multicolumn{3}{|c|}{$\begin{array}{l}\text { Dieta } \\
\text { Diet }\end{array}$} \\
\hline & $\begin{array}{l}\text { Controle } \\
\text { Control }\end{array}$ & $\begin{array}{c}250 \mathrm{~g} \\
\text { de óleo } \\
250 \text { g-oil }\end{array}$ & $\begin{array}{l}500 \mathrm{~g} \\
\text { de óleo } \\
500 \text { g-oi }\end{array}$ \\
\hline $\begin{array}{l}\text { Concentrado comercial }(\mathrm{kg} / \mathrm{dia}) \\
\text { Commercial concentrate }(\mathrm{kg} / \text { day })\end{array}$ & 4,90 & 4,10 & 3,10 \\
\hline Farelo soja $(\mathrm{kg} / \mathrm{dia})$ & 0,00 & 0,30 & 0,70 \\
\hline $\begin{array}{l}\text { Soybean meal }(\mathrm{kg} / \text { day }) \\
\text { Óleo }(\mathrm{kg} / \mathrm{dia}) \\
\text { Oil }(\mathrm{kg} / \text { day })\end{array}$ & 0,00 & 0,25 & 0,50 \\
\hline $\begin{array}{l}\text { Feno de coastcross }(\mathrm{kg} / \mathrm{dia}) \\
\text { Coastcross hay (kg/day) }\end{array}$ & 3,50 & 3,30 & 3,00 \\
\hline $\begin{array}{l}\text { Total }(\mathrm{kg} / \mathrm{dia}) \\
\text { Total }(\mathrm{kg} / \text { day })\end{array}$ & 8,40 & 7,95 & 7,30 \\
\hline \multicolumn{4}{|l|}{$\begin{array}{l}\text { Consumo de nutrientes } \\
\text { Nutrient intake }\end{array}$} \\
\hline $\begin{array}{l}\text { Matéria seca }(\mathrm{kg} / \mathrm{dia}) \\
\text { Dry matter (kg/day) }\end{array}$ & 7,94 & 7,44 & 6,83 \\
\hline $\begin{array}{l}\text { Matéria seca }(\mathrm{kg} / 100 \mathrm{~kg} \text { PC) } \\
\text { Dry matter }(\mathrm{kg} / 100 \mathrm{~kg} \mathrm{BW})\end{array}$ & 1,75 & 1,62 & 1,50 \\
\hline $\begin{array}{l}\text { Proteína bruta }(\mathrm{kg} / \mathrm{dia}) \\
\text { Crude protein }(\mathrm{kg} / \text { day })\end{array}$ & 0,93 & 0,92 & 0,91 \\
\hline $\begin{array}{l}\text { Energia digestível (Mcal/dia) } \\
\text { Digestible energy (Mcal/day) }\end{array}$ & 20,70 & 21,10 & 21,00 \\
\hline $\begin{array}{l}\text { Extrato étereo }(\mathrm{kg} / \mathrm{dia}) \\
\text { Ether extract }(\mathrm{kg} / \text { day })\end{array}$ & 0,16 & 0,36 & 0,62 \\
\hline $\begin{array}{l}\text { Cálcio (g/dia) } \\
\text { Calcium (g/day) }\end{array}$ & 49,56 & 43,53 & 35,88 \\
\hline $\begin{array}{l}\text { Fósforo }(\mathrm{g} / \text { dia }) \\
\text { Phosphorus (g/day) }\end{array}$ & 41,43 & 36,35 & 30,07 \\
\hline $\begin{array}{l}\text { Concentrado:volumoso } \\
\text { Concentrate:roughage ratio }\end{array}$ & $58: 42$ & $59: 41$ & $59: 41$ \\
\hline
\end{tabular}

vacuette, contendo FE sodiumFluoride/EDTA K3. Em seguida, o sangue foi armazenado a $-20^{\circ} \mathrm{C}$ e imediatamente encaminhado ao laboratório para as análises de glicose (glicose-Biobrás), lactato (Lactato-Sigma 735-10) e hematócrito.

Registraram-se as freqüências cardíaca e respiratória antes do início, após 1 e 2 horas de exercício e 15 minutos após o exercício. A freqüência cardíaca foi mensurada com estetoscópio no lado esquerdo dos animais e a freqüência respiratória, no terço superior da traquéia. A temperatura retal foi aferida no início e ao final do exercício-teste, utilizando-se termômetros da mesma marca, devidamente regulados. Todos os parâmetros fisiológicos e sangüíneos foram tomados ao mesmo tempo em todos os cavalos.

A temperatura ambiente, a umidade relativa do ar, a velocidade do vento e a precipitação pluviométrica no dia do exercício-teste (19/03/2003) foram obtidas na estação climatológica principal de Brasília (Inmet, 2003). Os valores 
Tabela 2 - Composição química dos componentes das dietas, com base na MS Table 2 - Chemical composition of components of the diets, on dry matter basis

\begin{tabular}{|c|c|c|c|c|}
\hline $\begin{array}{l}\text { Composição química } \\
\text { Chemical composition }\end{array}$ & $\begin{array}{l}\text { Concentrado comercial } \\
\text { Commercial concentrate }\end{array}$ & $\begin{array}{c}\text { Feno de coastcross } \\
\text { Coastcross hay }\end{array}$ & $\begin{array}{l}\text { Farelo de soja } \\
\text { Soybean meal }\end{array}$ & $\begin{array}{l}\text { Óleo de soja } \\
\text { Soybean oil }\end{array}$ \\
\hline $\operatorname{MS}(D M), \%^{1}$ & 93,57 & 95,92 & 88,56 & - \\
\hline $\mathrm{PB}(C P), \%^{1}$ & 17,39 & 3,92 & 46,93 & - \\
\hline $\mathrm{ED}(D E), \mathrm{kcal} / \mathrm{kg}^{2}$ & 3.289 & 1.682 & 3.494 & 9.000 \\
\hline FDN $(N D F), \%^{1}$ & 40,14 & 81,35 & 15,91 & - \\
\hline FDA $(A D F), \%^{1}$ & 14,00 & 43,72 & 10,32 & - \\
\hline $\operatorname{EE}(\%)^{1}$ & 2,69 & 1,02 & 1,88 & 99,99 \\
\hline MO $(O M), \%^{1}$ & 92,96 & 94,99 & 93,99 & - \\
\hline $\mathrm{Ca}(\%)^{1}$ & 0,81 & 0,37 & 0,28 & - \\
\hline $\mathrm{P}(\%)^{1}$ & 0,84 & 0,09 & 0,52 & \\
\hline
\end{tabular}

${ }_{1}^{1}$ Análises realizadas no Laboratório de Tecido Vegetal da Embrapa Cerrados, Brasília - DF.

2 Valores calculados de acordo com o NRC (1989).

1 Analyses were carried out at the Laboratory of Vegetable Tissue of Embrapa Cerrados, Brasilia - DF.

2 Value calculated according to NRC (1989).

de temperatura máxima, mínima e média foram 21,$8 ; 17,4$ e $19,3^{\circ} \mathrm{C}$, respectivamente, a umidade relativa do ar foi de $92 \%$, a velocidade do vento, $0,0 \mathrm{~m} / \mathrm{s}$ e a precipitação pluviométrica, $1,2 \mathrm{~mm}$.

Diariamente, foram coletadas amostras do feno de coastcross, do farelo de soja e da ração comercial, que formaram amostras compostas ao final dos 30 dias para análises dos teores de MS, nitrogênio, EE, Ca e P, realizadas conforme descrito por Silva (1990). As concentrações de FDN e FDA foram determinadas segundo metodologia descrita por Van Soest (1967) e Van Soest et al. (1991). Os resultados foram analisados pelo programa computacional, SAEG - Sistema de Análises Estatísticas e Genéticas (UFV, 1997).

\section{Resultados e Discussão}

Os teores de glicose, lactato e hematócrito no início e ao final do exercício-teste dos cavalos alimentados com as dietas experimentais são descritos na Tabela 3. Verificou-se efeito da interação $(\mathrm{P}<0,05)$ tempos de coleta $\times$ níveis de óleo na dieta sobre os valores de glicose, lactato e hematócrito. No início do exercício, os teores de glicose não foram afetados pelas dietas $(\mathrm{P}>0,05)$, porém, ao final do exercício, foi detectados valores mais elevados $(\mathrm{P}<0,05)$ nos cavalos que receberam diariamente 250 e $500 \mathrm{~g}$ de óleo na dieta. Estes resultados confirmam os achados de Hambleton et al. (1980), que verificaram correlação positiva do nível de glicose sangüínea com a quantidade de gordura na dieta. Cavalos alimentados com dieta contendo $16 \%$ de gordura apresentaram $58 \%$ mais glicose plasmática após o exercício que aqueles que receberam $4 \%$ de gordura na dieta. Resultados similares para os teores de glicose sangüínea após o exercício foram obtidos em cavalos submetidos a exercício-teste (galopes de $600 \mathrm{~m}$ ) após esforço físico com alta demanda anaeróbica (Oldaham et al., 1990); em cavalos alimentados duas vezes ao dia com $177 \mathrm{~mL}$ de óleo de milho na dieta submetidos a corridas de $1.600 \mathrm{~m}$; e em cavalos puro-sangue Inglês após alimentação com dieta contendo $400 \mathrm{~mL}$ de óleo de soja por períodos longos, de 7 a 8 meses (Pagan et al., 1995). Os cavalos alimentados e adaptados a dietas contendo óleo (250 e $500 \mathrm{~g}$ ) provavelmente aumentaram o metabolismo lipídico e a capacidade de oxidar ácidos graxos para utilização como fonte de energia, poupando as reservas de glicogênio e, conseqüentemente, disponibilizando maior quantidade de glicose sangüínea em relação àqueles alimentados com a dieta controle.

Entretanto, os dados da literatura são conflitantes quanto ao nível de glicose pós-exercício em cavalos alimentados com dieta contendo gordura animal ou vegetal. Taylor et al. (1995) e Frape (1994) constataram níveis mais elevados de glicose, enquanto Scott et al. (1992) e Marqueze et al. (2001) não observaram alterações nos níveis de glicose em cavalos exercitados a intensidades alta e média, respectivamente. Meyer et al. (1989) encontraram menores teores de glicose em cavalos submetidos a exercício-teste alimentados com dieta contendo $10 \%$ de gordura em comparação à dieta sem óleo.

Os teores de lactato no início do exercício-teste não foram influenciados pelas dietas $(\mathrm{P}>0,05)$, mas, ao final, os cavalos alimentados com as dietas contendo óleo (250 e $500 \mathrm{~g}$ ) apresentaram menores valores de lactato em comparação àqueles que receberam a dieta controle $(\mathrm{P}<0,05)$, os quais não diferiram entre si $(\mathrm{P}>0,05)$. Meyer et al. (1989) obtiveram resultados semelhantes, ou seja, constataram tendência de diminuição de lactato ao final do exercício em cavalos Quarto-de-Milha alimentados com dietas contendo de 5 a $10 \%$ de gordura. Os menores teores de lactato sangüíneo após o exercício nos cavalos que receberam óleo provavelmente estão relacionados à melhor condição fisio- 
Tabela 3 - Teores de glicose, lactato e hematócrito dos cavalos no início e ao final do exercício-teste Table 3 - Contents of glucose, lactate and haematocrit of horses in the starting and finishing test exercise

\begin{tabular}{|c|c|c|c|c|c|c|}
\hline \multirow[t]{2}{*}{$\begin{array}{l}\text { Ração } \\
\text { Diet }\end{array}$} & \multicolumn{2}{|c|}{$\begin{array}{c}\text { Glicose }^{1,2}(\mathrm{mg} / \mathrm{dL}) \\
\text { Glucose }^{1,2}(\mathrm{mg} / \mathrm{dL})\end{array}$} & \multicolumn{2}{|c|}{ 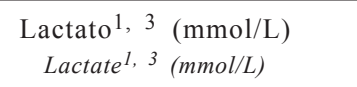 } & \multicolumn{2}{|c|}{$\begin{array}{c}\text { Hematócrito }^{1,} 4(\%) \\
\left.\text { Haematocrit }^{1,}, 4 \%\right)\end{array}$} \\
\hline & $\begin{array}{l}\text { Início } \\
\text { Before }\end{array}$ & $\begin{array}{l}\text { Final } \\
\text { After }\end{array}$ & $\begin{array}{l}\text { Início } \\
\text { Before }\end{array}$ & $\begin{array}{l}\text { Final } \\
\text { After }\end{array}$ & $\begin{array}{l}\text { Início } \\
\text { Before }\end{array}$ & $\begin{array}{l}\text { Final } \\
\text { After }\end{array}$ \\
\hline Controle (Control) & $99,4 \mathrm{Aa}$ & $104,8 \mathrm{Aa}$ & $0,58 \mathrm{Aa}$ & $5,73 \mathrm{Ba}$ & $32,1 \mathrm{Aa}$ & $61,0 \mathrm{Ba}$ \\
\hline $250 \mathrm{~g}$ óleo $(250 \mathrm{~g}$ oil $)$ & $98,0 \mathrm{Aa}$ & $127,0 \mathrm{Bb}$ & $0,52 \mathrm{Aa}$ & $2,92 \mathrm{Bb}$ & $35,8 \mathrm{Aab}$ & $57,0 \mathrm{Ba}$ \\
\hline $500 \mathrm{~g}$ óleo $(500 \mathrm{~g}$ oil $)$ & $105,0 \mathrm{Aa}$ & $133,0 \mathrm{Bb}$ & $0,54 \mathrm{Aa}$ & $2,67 \mathrm{Bb}$ & $38,8 \mathrm{Ab}$ & $50,0 \mathrm{Bb}$ \\
\hline
\end{tabular}

lógica desses animais durante o exercício, ocasionando atraso no limiar anaeróbico. Conseqüentemente, os ácidos graxos foram utilizados com fonte de energia para as células, poupando o glicogênio muscular e mantendo a glicose plasmática. No entanto, Oldham et al. (1990), Freitas et al. (2002) e Faustino et al. (2003), em estudo com cavalos alimentados com dieta contendo gordura ou óleo, verificaram tendência de níveis mais elevados de lactato após esforços de média e alta intensidade. Valores de lactato sem alteração durante ou após o exercício foram obtidos por Harkins et al. (1992), Scott et al. (1992) e Marqueze et al. (2001) em cavalos alimentados com óleo submetidos a exercícios de alta e média intensidade.

No início do exercício-teste, o valor do hematócrito foi menor nos cavalos alimentados com a dieta controle em relação aos do tratamento com $500 \mathrm{~g}$ de óleo por dia $(\mathrm{P}<0,05)$, que não diferiram daqueles que receberam a dieta contendo $250 \mathrm{~g}$ de óleo $(\mathrm{P}>0,05)$. Os valores de hematócrito ao final do exercício foram maiores que no início, independentemente da dieta $(\mathrm{P}<0,05)$, porém, os cavalos alimentados com a dieta contendo $500 \mathrm{~g}$ de óleo por dia apresentaram menores valores de hematócrito $(\mathrm{P}<0,05)$ ao término do exercício.

Estes resultados demonstram que o volume globular tende a acompanhar a intensidade do esforço atlético desenvolvido durante o exercício, ou seja, quanto maior o esforço, maior o volume globular. Contudo, menor elevação do hematócrito (11\%) foi detectada nos cavalos que receberam $500 \mathrm{~g}$ de óleo na dieta em relação àqueles dos tratamentos controle (29\%) e com 250 g de óleo ( $21 \%)$ ). O aumento do percentual eritrocitário e da hemoglobina circulante geralmente está correlacionado às dificuldades respiratórias e cardíacas, principalmente em razão da maior dificuldade de perfusão. No sangue mais viscoso, a velo- cidade de perfusão não acompanha as necessidades de oxigenação e de nutrientes no tecido da mesma forma que no sangue com hematócrito normal ou ligeiramente aumentado. Provavelmente os animais que receberam a dieta contendo $500 \mathrm{~g}$ de óleo apresentaram menor desidratação pós-exercício (principalmente em razão da temperatura corpórea mais baixa) que os do tratamento controle e, conseqüentemente, ocorreu menor taxa de hemoconcentração.

Hematócrito de 40 a $50 \%$ para um cavalo em exercício, conforme constatado nos cavalos que receberam $500 \mathrm{~g}$ de óleo, é indicativo de que o sangue teve concentração de hemoglobina suficiente para transportar oxigênio sem prejudicar o trabalho do sistema cardiorespiratório. Estes resultados divergem dos obtidos por Kurcz et al. (1993), que não detectaram alteração no hematócrito e nas concentrações sangüíneas em cavalos sob suplementação com óleo. Hambletton et al. (1980), em estudo utilizando dietas isocalóricas $(4,8,12$ e 16\% de gordura), também não notaram efeito da dieta sobre a hemoglobina ou o volume celular.

Os valores de temperatura corporal no início e ao final do exercício são descritos na Tabela 4. Observou-se interação tempo de tomada da temperatura $\times$ níveis de adição de óleo na ração $(\mathrm{P}<0,05)$. A temperatura corporal no início do exercício não foi afetada pelos níveis de óleo na $\operatorname{dieta}(\mathrm{P}>0,05)$, mas, ao final, verificaram-se menores valores de temperatura nos cavalos que receberam diariamente 250 e $500 \mathrm{~g}$ de óleo na dieta $(\mathrm{P}<0,05)$, que não diferiram entre si $(\mathrm{P}>0,05)$.

Segundo Potter et al. (1990), o fornecimento de dieta suplementada com óleo diminui o estresse termal, ou seja, o incremento calórico. Scott et al. (1993) verificaram redução de $14 \%$ na produção de calor corporal em cavalos da raça puro-sangue Inglês submetidos ao exercício quando 
Tabela 4 - Temperatura corporal dos cavalos no início e ao final do exercício-teste

Table 4 - Body temperature of horses in the beginning and end of test exercise

\begin{tabular}{|c|c|c|}
\hline \multirow[t]{2}{*}{$\begin{array}{l}\text { Ração } \\
\text { Diet }\end{array}$} & \multicolumn{2}{|c|}{$\begin{array}{c}\text { Temperatura corporal }\left({ }^{\circ} \mathrm{C}\right)^{1,}, 2 \\
\text { Body temperature }\left({ }^{\circ} \mathrm{C}\right)^{1,2}\end{array}$} \\
\hline & $\begin{array}{l}\text { Início } \\
\text { Before }\end{array}$ & $\begin{array}{l}\text { Final } \\
\text { After }\end{array}$ \\
\hline Controle (Control) & $37,45 \mathrm{Aa}$ & $40,00 \mathrm{Ba}$ \\
\hline $250 \mathrm{~g}$ óleo $(250 \mathrm{~g}$ oil $)$ & $37,62 \mathrm{Aa}$ & $39,28 \mathrm{Bb}$ \\
\hline $500 \mathrm{~g}$ óleo $(500 \mathrm{~g}$ oil $)$ & $37,57 \mathrm{Aa}$ & $39,22 \mathrm{Bb}$ \\
\hline
\end{tabular}

${ }^{1}$ Médias seguidas de pelo menos uma mesma letra maiúscula na linha não diferem $(P>0,05)$ pelo teste $F$ e de pelo menos uma mesma letra minúscula na coluna não diferem $(P>0,05)$ pelo teste Tukey.

${ }^{2} \mathrm{CV}_{2}=1,01 \%$

1 Means followed by the same capital letter in a row do not differ $(P>0.05)$ by F test and small letter in a column do not differ $(P>0.05)$ by Tukey test.

${ }^{2}$ Coefficient of variation (CV) $-\mathrm{CV}_{2}=1.01 \%$.

acrescentado óleo à ração. Além disso, a inclusão de óleo na dieta pode favorecer o desempenho de cavalos exercitados em regiões de clima quente e úmido, em razão da diminuição do incremento calórico, pois aproximadamente $3 \%$ a mais de calor é produzido durante a formação de ATP via oxidação da glicose, quando comparado à oxidação dos ácidos graxos (Konh et al., 1996). O menor valor da temperatura corporal ao final do exercício nos cavalos que receberam óleo provavelmente esteja relacionado à menor fermentação no intestino e ao menor incremento calórico deste alimento, facilitando a manutenção mais eficiente da temperatura corporal dos cavalos. No entanto, Freitas et al. (2002) não verificaram diferenças na temperatura corporal, após prova de enduro de $30 \mathrm{~km}$, de cavalos Mangalarga Marchador alimentados com dieta contendo 0 e $27,5 \%$ de óleo.

Os resultados das freqüências cardíacas antes, durante e após o exercício são descritos na Tabela 5. Foi detectada interação períodos de tomada da freqüência cardíaca $\times$ níveis de óleo na ração $(P<0,05)$. No início do exercício, não houve influência das dietas na freqüência cardíaca $(\mathrm{P}>0,05)$, mas, ao final ( 2 horas de exercício) e 15 minutos após o término da prova, os cavalos que receberam $500 \mathrm{~g}$ de óleo por dia apresentaram menores valores de freqüência cardíaca que aqueles das demais dietas $(\mathrm{P}<0,05)$. Esses resultados divergem dos encontrados por Marqueze et al. (2001) e Freitas et al. (2002), que não detectaram influência da dieta contendo óleo sobre a freqüência cardíaca antes e após o exercício em cavalos exercitados a média e alta intensidade.

O maior valor de freqüência cardíaca aos 15 minutos após o exercício no grupo de cavalos que receberam a dieta controle provavelmente esteja relacionado à tentativa do organismo animal em manter a termorregulação e oferecer
Tabela 5 - Freqüência cardíaca dos cavalos no início, durante (1 e 2 horas) e 15 minutos após o término do exercícioteste

Table 5 - Heart rate of horses in the beginning, at 1 and 2 hours and 15 minutes post test exercise

\begin{tabular}{|c|c|c|c|c|}
\hline \multirow[t]{2}{*}{$\begin{array}{l}\text { Dieta } \\
\text { Diet }\end{array}$} & \multicolumn{4}{|c|}{$\begin{array}{c}\text { Freqüência cardíaca (batimentos } / \text { minuto) })^{1,4} \\
\text { Heart rate (beats/minutes) } 1,4\end{array}$} \\
\hline & $\begin{array}{l}0 \text { hora } \\
0 \text { hour }\end{array}$ & $\begin{array}{l}1 \text { hora } \\
1 \text { hour }\end{array}$ & $\begin{array}{ll}2 & \text { horas } \\
2 & \text { hours }\end{array}$ & $\begin{array}{l}15 \text { minutos após } \\
15 \text { minutes after }\end{array}$ \\
\hline $\begin{array}{l}\text { Controle }{ }^{2} \\
\text { Control }\end{array}$ & $35,00 \mathrm{a}$ & $72,17 \mathrm{a}$ & $108,33 \mathrm{a}$ & $103,00 \mathrm{a}$ \\
\hline $\begin{array}{l}250 \mathrm{~g} \mathrm{óleo}^{3} \\
250 \mathrm{~g} \text { oil }\end{array}$ & $29,67 \mathrm{a}$ & $86,83 \mathrm{a}$ & $97,00 \mathrm{a}$ & $59,00 \mathrm{~b}$ \\
\hline $\begin{array}{l}500 \mathrm{~g} \mathrm{óleo} \\
500 \mathrm{~g} \text { oil }\end{array}$ & $30,00 \mathrm{a}$ & $76,83 \mathrm{a}$ & $68,67 b$ & $38,33 \mathrm{c}$ \\
\hline
\end{tabular}

${ }^{1}$ Médias seguidas de pelo menos uma mesma letra na coluna não diferem $(P>0,05)$ pelo teste Tukey.

2 Efeito linear na linha $(P<0,01)$

3 Efeito quadrático na linha $(P<0,01)$

${ }^{4} \mathrm{CV}_{2}=12,13 \%$

1 Means followed by the same letter in a column do not differ $(P>0.05)$ by Tukey test.

${ }^{2}$ Linear effect in the row $(P<0.01)$.

${ }^{3}$ Quadratic effect in the row $(P<0.01)$.

${ }^{4}$ Coefficient of variation (CV) - $C V_{2}=12.3 \%$.

melhor aporte de oxigênio à musculatura. No entanto, os grupos que receberam $250 \mathrm{e} 500 \mathrm{~g}$ de gordura mantiveram a temperatura corporal mais baixa, de modo que o sistema cardiorespiratório não foi sobrecarregado para dissipar o excesso de calor, mantendo a freqüência cardíaca em valores mais baixos em relação à observada com a dieta controle. O hematócrito dos cavalos que receberam 500 g de óleo por dia proporcionou a esses animais melhor desempenho circulatório, em decorrência da menor viscosidade do sangue e da maior velocidade de perfusão, não sobrecarregando o coração.

Os valores de freqüência respiratória observados no início, durante e após o exercício dos cavalos alimentados com as três dietas são apresentados na Tabela 6. Foi constatada interação $(\mathrm{P}<0,05)$ tempos de tomada da freqüência respiratória $\times$ tratamentos. No início e ao final do exercício ( 2 horas), não foram detectadas diferenças nas freqüências respiratórias entre os tratamentos $(\mathrm{P}>0,05)$, no entanto, com 1 hora de exercício os cavalos alimentados com a dieta controle apresentaram menor freqüência respiratória, seguidos dos alimentados com a dieta contendo $250 \mathrm{~g}$ de óleo, que, por sua vez, foram seguidos daqueles que consumiram $500 \mathrm{~g}$ de óleo por dia $(\mathrm{P}<0,05)$. Quinze minutos após o exercício, os cavalos alimentados com as dietas contendo óleo apresentaram melhores valores de freqüência respiratória $(\mathrm{P}<0,05)$ em relação àqueles que receberam a dieta controle.

A melhor recuperação respiratória verificada nos cavalos que receberam óleo se deve, provavelmente, ao fato de que a oxidação dos ácidos graxos produz menos dióxido de 
Tabela 6 - Freqüência respiratória dos cavalos no início, durante (1 e 2 horas) e 15 minutos após o término do exercícioteste

Table 6 - Respiratory frequency pre, during (1 and 2 hours) and 15 minutes post exercise test

\begin{tabular}{|c|c|c|c|c|}
\hline \multirow[t]{2}{*}{$\begin{array}{l}\text { Dieta } \\
\text { Diet }\end{array}$} & \multicolumn{4}{|c|}{$\begin{array}{c}\text { Freqüência respiratória (respirações } / \text { minuto) }{ }^{1,3} \\
\text { Respiratory rate }(\text { Respiration } / \text { minute) })^{1,3}\end{array}$} \\
\hline & $\begin{array}{l}0 \text { hora } \\
0 \text { hour }\end{array}$ & $\begin{array}{l}1 \text { hora } \\
1 \text { hour }\end{array}$ & $\begin{array}{l}2 \text { horas } \\
2 \text { hours }\end{array}$ & $\begin{array}{l}15 \text { minutos após } \\
15 \text { minutes after }\end{array}$ \\
\hline $\begin{array}{l}\text { Controle }^{2} \\
\text { Control }\end{array}$ & $11,33 \mathrm{a}$ & $37,67 \mathrm{a}$ & $65,50 \mathrm{a}$ & $47,83 \mathrm{a}$ \\
\hline $\begin{array}{l}250 \mathrm{~g}^{\text {óleo }}{ }^{2} \\
250 \mathrm{~g} \text { oil }\end{array}$ & $12,00 \mathrm{a}$ & $45,33 b$ & $59,50 \mathrm{a}$ & $21,00 \mathrm{~b}$ \\
\hline $\begin{array}{l}500 \mathrm{~g} \text { óleo } \\
500 \mathrm{~g} \text { oil }\end{array}$ & $10,83 a$ & $66,55 \mathrm{c}$ & $62,83 a$ & $23,83 b$ \\
\hline
\end{tabular}

${ }^{1}$ Médias seguidas de pelo menos uma mesma letra na coluna não diferem $(P>0,05)$ pelo teste Tukey.

2 Efeito quadrático na linha $(P<0,01)$.

${ }^{3} \mathrm{CV}_{2}=12,88 \%$.

1 Means followed by the same letter in a column do not differ $(P>0.05)$ by Tukey test.

${ }^{2}$ Quadratic effect in the row $(P<0.01)$

${ }^{3}$ Coefficient of variation (CV) $-C_{2}=12.88 \%$

carbono que a oxidação da glicose, reduzindo, portanto, o esforço respiratório (Holloszy et al., 1986; Frape, 1994). Possivelmente, o elevado valor da freqüência respiratória aos 15 minutos pós-exercício nos cavalos que receberam a dieta controle esteja relacionado à redução da capacidade de carreamento de oxigênio, visto que o sangue destes animais tornou-se mais viscosos e com menor oxigenação tecidual, como resultado da intensificação das atividades cardíaca e respiratória, principalmente pela tentativa do organismo em manter a temperatura corporal constante.

\section{Conclusões}

A adição de 250 e $500 \mathrm{~g}$ de óleo na dieta de eqüinos submetidos a exercício de média intensidade proporcionou melhora no desempenho hematofisiológico e, portanto, pode resultar em melhor desempenho atlético aos cavalos. Os cavalos que consumiram $500 \mathrm{~g}$ de óleo por dia apresentaram melhor recuperação pós-prova, confirmada pela freqüência cardíaca e pelos resultados do hematócrito.

\section{Literatura Citada}

CUNHA, T.J. Horse feeding and nutrition. 2.ed. San Diego: Academic Press, 1991. 445p.

FAUSTINO, M.G.; OLIVEIRA, K.; SANTOS, V. P. et al. Efeito da adição de óleo de soja sobre as concentrações bioquímicas de cavalo de enduro. In: CONGRESSO INTERNACIONAL DE ZOOTECNIA, 5., 2003, Uberaba. Anais... Uberaba: Associação Brasileira de Zootecnia, 2003. v.2, p.175-178.

FERRANTE, P.L.; TAYLOR, L.E.; KRONFELD, D. et al. Blood lactate concentration during exercise in horse fed a high-fat diet an administered sodium bicarbonate. Journal of Nutrition, v.124, p.2738-2739, 1994.

FRAPE, D.L. Diet and exercise performance in the horse. Proceedings of the Nutrition Society, v.53, p189-206, 1994

FREITAS, E.V.V.; CARVALHO, G.R.; FORTES, C.M.L.S. et al. Adição de óleo na dieta de eqüinos de raça Mangalarga Marchador, submetidos à provas de resistência. In: REUNIÃO ANUAL DA SOCIEDADE BRASILEIRA DE ZOOTECNIA, 39. 2002, Recife. Anais... Recife: Technomedia, 2002 (CD-ROM). Nutrição de Não-Ruminantes.

HAMBLENTON, P.L.; SLADE, L.M.; HAMAR, D.W. et al. Dietary fat and exercise conditioning effect on metabolic parameters in the horse. Journal of Animal Science, v. 51, n.6, p.1330-1339, 1980 .

HARKINS, J.D.; MORRIS, G.S.; TULBY, R.T. et al. Effect of added fat on racing performance in thoroughbred horse. Journal Equine Veterinary Science, v.12, n.2, p.123-129, 1992.

HINEY, K.M.; POTTER, G.D. A review of recent research on nutrition and metabolism in the athletic horse. Nutrition Research Reviews, v.9, p.149-173, 1996.

HINTZ, H.F. Alimentado o cavalo atleta. In: SIMPÓSIO INTERNACIONAL DO CAVALO DE ESPORTE, 1997, Belo Horizonte. Anais... Belo Horizonte: Cadernos Técnicos da Escola de Veterinária da UFMG, 1997, n.19, p.49-58.

HINTZ, H.F. Nutrition and equine performance. Journal of Nutrition, v.124, n.12, p.2723-2729, 1994

HOLlOSZY, J.O.; DALSKY, G.P.; NEMETH, P.M. et al. Muscle triglyceride utilization during exercise: effect of training. Journal Applied Physiology, v.60, p.562, 1986.

INMET [2003]. Disponível em: www.inmet.go.br Acesso em: $15 / 04 / 2003$.

JONES, D.L.; POTTER, G.D.; GREENE, L.W. et al. Muscle glycogen in exercised miniature horses at various body conditions an fed a control or fat supplemented diet. Journal Equine Veterinary Science, v.12, p.287-291, 1992.

KOHN, C.; ALLEN, A.K.; HARRIS, P. et al. Nutrition for the equine athlete. The Equine Athlete, v.9, n.4, p.12-17, 1996.

KRONFELD, D.S.; CRANDEL, K.M.; CUSTALOW, S.E. et al. Studies of fat adaptation and exercise. In: RECENT ADVANCES IN EQUINE NUTRITION, 1998, Kentucky. Proceedings... Kentucky: Kentucky Equine Research, 1998. p.37-39.

KURCZ, E.V.; SCURG, W.A; MARCHELLOB, J.A. et al. Dietary fat supplementation changes in lipoprotein composition in horses. In: EQUINE NUTRITION AND PHYSIOLOGY SOCIETY, 13., 1993, Gainesville. Proceedings... Gainesville: The Equine Nutrition and Physiology Society, 1993. p.167-172.

LAWRENCE, L.M.; HINTZ, H.F.; SODERHOLM, L.V. et al. Effect of time of feeding on metabolic response to exercise. Journal Equine Veterinary Science, v.18, p.392-395, 1995.

MARQUEZE, A.; KESSLER, A.M.; BERNADI, M.L. Aumento do nível de óleo em dietas isonergéticas para cavalos submetidos a exercício. Ciência Rural, v.31, p.491-496, 2001.

MEYER, M.C.; POTTER, G.D.; EVANS, J.W. et al. Physiologic and metabolic response of exercising horse fed added dietary fat. Journal Equine Veterinary Science, v.9, n.4, p218223, 1989.

NATIONAL RESEARCH COUNCIL - NRC. Nutrient requirements of horses. 5.ed.rev. Washington, D.C.: National Academy Press, 1989. 100p.

OLDHAM S.L.; POTTER, G.D.; EVANS, J.W. et al. Storage and mobilization of muscle glycogen in exercising horse fed a fat supplemented diet. Journal Equine Veterinary Science, v. 10, n.5, p.353-359, 1990 .

PAGAN, J.D.; ROTMENSE, T.; JACKSON, S.G. Responses of blood glucose, lactate and insulin in horses fed equal amounts of grain with or without added soybean oil. In: RECENT ADVANCES IN EQUINE NUTRITION, 1995, Kentucky. Proceedings... Kentucky: Kentucky Equine Research, 1995. p.49-56. 
PAGAN, J.D. Time of feeding critical for performance. In: ADVANCES IN EQUINE NUTRITION, 1999, Kentucky. Proceedings... Kentucky: Kentucky Equine Research, 1999. p.117-126.

POTTER, G.D.; WEBB, S.P.; EVANS, J.W. et al. Digestible energy requirements for work and maintenance of horses fed conventional and fat-supplemented diets. Journal Equine Veterinary Science, v.3, p.214-218, 1990.

SCOTT, B.D.; POTTER, G.D. GREEN, L.W. et al. Efficacy of a fat supplemented diet on muscle glycogen concentration in exercising thoroughbred horses maintained in varying body condition. Journal Equine Veterinary Science, v.12, p.109113, 1992.

SCOTT, B.D.; POTTER, G.D.; GREEN, L.W. et al. Efficacy of a fat supplemented diet to reduce thermal stress in exercising thoroughbred horses. In: EQUINE NUTRITION AND PHYSIOLOGY SOCIETY, 13., 1993, Gainesville. Proceedings... Gainesville: The Equine Nutrition and Physiology Society, 1993. p.66-71.

SILVA, D.J. Análise de alimentos: métodos químicos e biológicos. 2.ed. Viçosa, MG: Universidade Federal de Viçosa, 1990. 165p.
TAYLOR, L.E.; FERRANTE, P.L.; KRONFELD, D.S. et al. AcidBase variables during incremental exercise in sprint-trained horses fed a high-fat diet. Journal of Animal Science, v.73, p.2009-2018, 1995.

UNIVERSIDADE FEDERAL DE VIÇOSA - UFV. SAEG - Sistema para análises estatísticas e genética. Versão7.1. Viçosa, MG: 150p (Manual do usuário).

Van SOEST, P.J. Development of a comprehensive system of feed analyses and its application to forages. Journal of Animal Science, v.26, n.1, p.119-128, 1967.

Van SOEST, P.J., ROBERTSON, J.B., LEWIS B.A. Methods for dietary fiber, neutral detergent fiber, and nonstrach polysaccharides in relation to animal nutrition. Journal of Dairy Science, v.74, n.4, p.3583-3597, 1991. 